\title{
Relaxation of the Elastic Clamp in Rail Fastenings
}

\section{Maxim Arbuzov, Serhii Tokariev, Oleksii Hubar, Volodymyr Andrieiev, Volodymyr Suslov}

Department of Transport Infrastructure, Dnipro National University of Railway Transport named after Academician V. Lazaryan, Lazaryan st. 2, 49010, Dnipro, Ukraine; \{ maximarbuzov, serhiitokariev, oleksiihubar, volodymyrandrieiev $\}$ @ diit.edu.ua Company LLC Research and Production Enterprise "KRT CORPORATION", Komarnivska st. 66, 81500, Gorodok city, Gorodotskiy district, Lviv region, Ukraine; spc@krt.co.ua

\begin{abstract}
The main purpose of a rail fastening system is to provide the strength and reliability of joining rails to sleepers and, thus, prevents the longitudinal rail displacement and track disturbance. In a railway fastening, the joining function is performed by an elastic clamp. Over time, the clamping force of the elastic clamp is decreased due to various factors. The elastic clamp relaxation phenomenon is studied in present work. The observation of the clamp residual deformation was carried out on predetermined sleepers in different operating conditions. According to the research results of the elastic clamp relaxation of the rail fastenings, the following conclusions can be made: there is a relaxation process in the elastic clamps of the rail fastening, which has a curvilinear dependence on time; the intensity of the elastic clamps relaxation attenuates after one year of operation; in addition to the time factor, the relaxation of elastic clamps is influenced by the level of operating stresses; greater value of operating stresses corresponds to greater relaxation.
\end{abstract}

\section{The Research Program}

Today in the countries of the European space [1] the design of elastic intermediate rail fastening is used, which combines all advanced achievements. Elastic fastenings are manufactured in many countries around the world. In order for this work not to be an advertisement, this paper does not indicate the type of rail fastening. Elastic fastening has different design options. But all elastic fastenings have a common element. This is an elastic clamp. The elastic clamp is the main element in rail fastening system. The elastic clamp must work for a long time. 
The present work, studies the behavior of elastic fastening over time. Elastic fastening in its design includes elastic clamps that press a rail to a sleeper. In a free state, the elastic clamp has initial sizes. During mounting of the clamp, elastic deformations and mounting mechanical stresses occur. This creates a force that presses the rail to the sleeper. The mechanical properties of elastic clamps change over time. The reducing phenomenon of elastic deformations and mounting mechanical stresses is called relaxation. During relaxation of the elastic clamps, part of the elastic deformation transforms into plastic. As a result, the clamping force of the rail to the sleeper is decreased. At low values of the clamping force, the rail track malfunctions and the level of train safety decreases.

The research object is the elastic clamps of the intermediate rail fastening. The aim of present work is to research the effect of time on the geometric sizes of elastic clamps installed in a railway track.

An elastic rail fastening that is in operation is subject to research. When studying the relaxation of elastic clamps, it is necessary to establish how different factors affect. Experimental elastic fastening is affected not only by time but also the force effect from the wheels of the rolling stock. It is also necessary to determine how the track horizontal curvature affects the rail track, how the dismantling of the elastic clamp itself affects. It is necessary to determine whether the temperature affects the deformation of the elastic clamp.

The answer to the question "affects or does not affect" can be got by a special experiment or by factor analysis. Factor analysis of variance is performed under the Regulations on the procedure for conducting acceptance tests of railway track superstructure element prototypes [2, 3].

Analysis of variance task is to compare the estimation of the variance caused by the influence of a factor with the estimation of the variance caused by the influence of a random component. Comparing this relationship with Fisher's criterion $[F]$, a conclusion is made about the influence of the factor on the study parameter [4]. If the calculated value of the criterion $F$ more than $[F]$, the factor affects. If the calculated value of the criterion $F$ less than $[F]$, the factor is not affected.

Object No. 1. To study the relaxation process of the elastic clamp, a test section of the track in operation was selected. A new elastic fastening was placed on this section of the track, which was monitored. Measurements were periodically performed at the test section to accumulate passed tonnage of over 100 million tons, which is 1 year and 4 months. These elastic clamps were affected by all the factors listed in paragraph 1.2.1.

Object No. 2. A rail-and-sleeper grid with a new fastening was also assembled and placed outside of the track. The movement of trains on it was not carried out. The rail-and-sleeper grid is straight. The elastic fastening of the rail-and-sleeper grid was not affected by the rolling stock. The study of this fastening was carried out simultaneously with the fastening that was in operation (object No. 1). 
Table 1

Frequency of measurements of elastic clamps

\begin{tabular}{|c|c|c|c|}
\hline $\begin{array}{c}\text { Measurement } \\
\text { number }\end{array}$ & Date & $\begin{array}{c}\text { Operation time, } \\
\text { months }\end{array}$ & $\begin{array}{c}\text { Passed tonnage at the time of } \\
\text { measurement, million tons gross }\end{array}$ \\
\hline 0 & August 2018 & 0 & 0 \\
\hline 1 & November 2018 & 3 & 21.96 \\
\hline 2 & March 2019 & 7 & 49.10 \\
\hline 3 & June 2019 & 10 & 72.30 \\
\hline 4 & December 2019 & 16 & 107.01 \\
\hline
\end{tabular}

Objects No. 3 and No. 4. To study the relaxation process over a longer period of time, additional control track sections were selected, which worked for a long time. The control sections are sections of the same track construction as the test section (object No. 1), the operating conditions of which (a radius, passed tonnage, an axial load) do not differ by more than $10 \%$ from the operating conditions of the test facility (object No. 1), and a load intensity differ by at least $50 \%$ and $75 \%$. In this case, the operating conditions of the elastic fastening of objects No. 1, No. 3, No. 4 differ only in the operating time. One-time measurements were performed at the control sections.

An information search in literary sources was also conducted about the elastic fastenings which worked over a long period of time. Periodically at the test section and once at the control sections evaluations were conducted monitoring the failure of the fastening units and observation of residual deformation of the elastic clamps.

The observation of the clamp residual deformation was carried out on predetermined sleepers - control cross sections. The control cross sections were located in the middle of the circular curves and on the straight section of the track. The distance between the control cross sections is approximately $10 \mathrm{~m}$ from each other. The clamps did not replace during the research period.

During the observation, the actual geometric sizes of the elastic clamps were determined and compared with their initial values. Thus, the plastic deformation of the elastic clamp was determined, which reflects the relaxation process. The initial values of the geometric sizes of the elastic clamps were obtained during measurements taken before laying into the track.

Dismantling of the fastening unit was carried out by by the track facility workers, measurements were performed by scientists of the Dnipro National University of Railway Transport named after Academician V. Lazaryan.

The elastic clamp of the rail fastening has a symmetrical curvilinear shape. One part of the clamp is rigidly attached to the sleeper, and the other elastically presses on the rail. The elastic clamp is dismantled and the sizes " $\mathrm{S}$ " and " $\mathrm{Z}$ " are measured in the free state (Fig. 1). Measurements are performed with a caliper with an accuracy of $0.1 \mathrm{~mm}$. An auxiliary equipment is also used for measurement. 


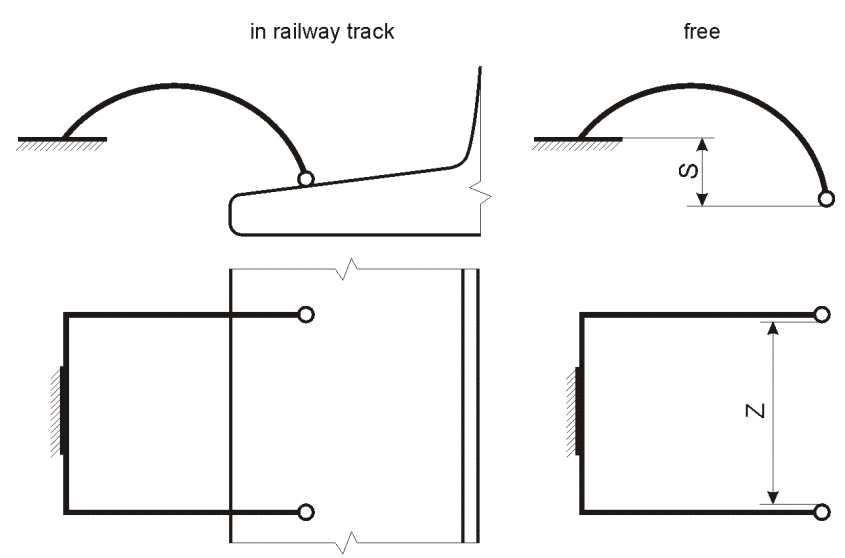

Figure 1

The measurement schema of the size "S" and the size " $\mathrm{Z}$ " of the elastic clamp

The residual deformation of the elastic clamp was estimated by changing the sizes "S" and "Z" according to formula (1) and (2), respectively.

$\Delta S=S_{\mathrm{n}}-S_{0}$

where $\Delta S$ is the vertical residual deformation of the elastic clamp;

$S_{\mathrm{n}}$ is the size "S" during the $n$-th dismantling of the elastic clamp for measurements;

$S_{0}$ is the size "S" before the initial laying of the elastic clamp into the track.

$\Delta Z=Z_{\mathrm{n}}-Z_{0}$

where $\Delta Z$ is the horizontal residual deformation of the elastic clamp;

$Z_{\mathrm{n}}$ is the size " $Z$ " during the $n$-th dismantling of the elastic clamp for measurements;

$Z_{0}$ is the size " $Z$ " before the initial laying of the elastic clamp into the track.

\section{Analysis of Research Data}

The track facility workers observed the elastic fastening operation every day. Scientists periodically conducted a control inspection when measuring the elastic clamps. The frequency of follow-up surveys was once every three months. During the entire period of operational tests, the elastic clamp failures due to passed tonnage or high load intensity of the section were absent. Failures of other fasteners during operational tests were also not observed. 
Measurements of elastic clamps were carried out after their dismantling. The elastic clamps were in a free state. After measurements, the elastic clamp was mounted into the track in its place. It worked for some time and was dismantled again during subsequent measurements. In such studies, it is important to know whether the dismantling process itself affects the elastic clamp. To answer this question, the following experiment was conducted. Dismantled the elastic clamp. Size "S" and size " $Z$ " were measured. Mounted the elastic clamp in its place. Dismantled the elastic clamp again. Size "S" and size " $Z$ " were measured again. This was repeated five times. Size "S" and size " $Z$ " have not changed. Therefore, the dismantling of the elastic clamp does not affect its residual deformation.

Measurements of elastic clamps were carried out at different ambient temperatures. Measurements were performed in August, November, March, June, and December. There were positive and negative temperatures. It is known that when heated, the metal expands. The elastic clamp has internal stresses from its production. It is important to know how temperature affects the deformation of the elastic clamp. The following experiment was performed to answer this question. Dismantled the elastic clamp. Size "S" and size "Z" were measured. The temperature of the elastic clamp was measured. The elastic clamp was heated to $15^{\circ} \mathrm{C}$. Size " $\mathrm{S}$ " and size " $Z$ " were measured again. The elastic clamp was heated to another $15^{\circ} \mathrm{C}$. This was repeated five times. Size "S" and size " $Z$ " have not changed. Therefore, the change in temperature of the elastic clamp does not cause its deformation. The effect of seasonal temperature fluctuations was also not detected.

The influence of the track radius on the residual deformation of the elastic clamp is estimated from the measurements of the object No. 1 via one-factor analysis. The elastic clamps at object No. 1 were located in the straight section of the track, in a curve with a radius of $874 \mathrm{~m}$ and in a curve with a radius of $1062 \mathrm{~m}$. Therefore, three levels of factor were adopted for one-factor analysis.

Table 2 shows the results of one-factor analysis for the significance level $\alpha=0.05$. Calculations were made for the different passed tonnage. The results for passed tonnage equal to zero are not shown, as the elastic clamps have not yet been mounted into the rail track. The results show that at the beginning of operation the track radius has a negligible effect on the geometric sizes of the elastic clamp. Then this effect disappears. This phenomenon is called "adaptation".

Passed tonnage influence on the elastic clamp residual deformation is estimated from the measurements of the object No. 1 via one-factor analysis. The elastic clamps at object No. 1 were measured five times. Therefore, five factor levels were adopted for one-factor analysis. Table 3 shows the results of one-factor analysis for the significance level $\alpha=0.05$. The results show that passed tonnage is a very influential factor. 
Table 2

One-factor analysis results of the track radius influence on the residual deformation of the elastic clamp

\begin{tabular}{|c|c|c|c|c|c|c|}
\hline $\begin{array}{c}\text { Passed } \\
\text { tonnage, } \\
\text { million } \\
\text { tons gross }\end{array}$ & \multicolumn{3}{|c|}{ for size "Z" } & \multicolumn{3}{c|}{ for size "S" } \\
\cline { 2 - 7 } & $F$ & {$[F]$} & conclusion & $F$ & {$[F]$} & conclusion \\
\hline 21.96 & 4.284 & 3.074 & affects & 0.816 & 3.074 & does not affect \\
\hline 49.10 & 0.198 & 3.074 & does not affect & 3.514 & 3.074 & affects \\
\hline 72.30 & 2.361 & 3.074 & does not affect & 0.850 & 3.074 & does not affect \\
\hline 107.01 & 0.886 & 3.074 & does not affect & 1.925 & 3.074 & does not affect \\
\hline
\end{tabular}

Table 3

The results of one-factor analysis of the passed tonnage influence on the residual deformation of the elastic clamp

\begin{tabular}{|c|c|c|c|c|c|}
\hline \multicolumn{3}{|c|}{ for size "Z" } & \multicolumn{3}{c|}{ for size "S" } \\
\hline$F$ & {$[F]$} & conclusion & $F$ & {$[F]$} & conclusion \\
\hline 62.467 & 2.387 & affects & 46.190 & 2.387 & affects \\
\hline
\end{tabular}

It is known that the dynamics of the track and rolling stock interaction in the straight section and in curve differ. In the curved section of the track there is a centrifugal horizontal force, which is transmitted through the rail to the fastenings. Simultaneous influence of two factors - radius and passed tonnage - is investigated by two-factor analysis, the results of which for the significance level $\alpha=0.05$ are shown in Table 4 . The results show that the simultaneous influence of the radius and passed tonnage has a minor effect on geometric sizes of the elastic clamps.

Table 4

The results of a two-factor analysis of the simultaneous effect of the curve radius and passed tonnage on the residual deformation of the elastic clamp

\begin{tabular}{|c|c|c|c|c|c|}
\hline \multicolumn{3}{|c|}{ for size "Z" } & \multicolumn{3}{c|}{ for size "S" } \\
\hline$F$ & {$[F]$} & conclusion & $F$ & {$[F]$} & conclusion \\
\hline 2.645 & 1.954 & affects & 2.518 & 1.954 & affects \\
\hline
\end{tabular}

The observation results of the horizontal residual deformation $\Delta Z$ of the elastic clamps of the object No. 1 are shown in Figure 2. The data for the straight section of the track is shown here so it can be compared with the data for the section No. 2. The points on the graph show the average values of measurements at different passed tonnage. The obtained data are approximated by the function $\Delta Z=A \cdot \operatorname{atan}(B \cdot T)$, where $A$ and $B$ are constant approximations: $A=0.9 ; B=0.05$. Along the ordinate axis is the residual deformation $\triangle Z$. Passed tonnage $T$ is on the abscissa. 
The results show that at the beginning of operation passed tonnage has a significant effect on the size " $Z$ " of the elastic clamp. Then this effect fades. The results of the relaxation intensity, which fades over time, is confirmed by the classical mechanics [5].

The observation results of the vertical residual deformation $\Delta S$ of the elastic clamps of the object No. 1 are shown in Figure 3. The data for the straight section of the track is shown here so it can be compared with the data for the section No. 2. The obtained data are approximated by the function $\Delta S=C \cdot \operatorname{atan}(D \cdot T)$, where $C$ and $D$ are constant approximations: $C=0.6 ; D=0.05$. On the y-axis - the residual deformation $\Delta S$. The abscissa axis is the paseed tonnage $T$. The results show that at the beginning of operation the paseed tonnage has a significant effect on the size "S" of the elastic clamp. Then this effect fades.

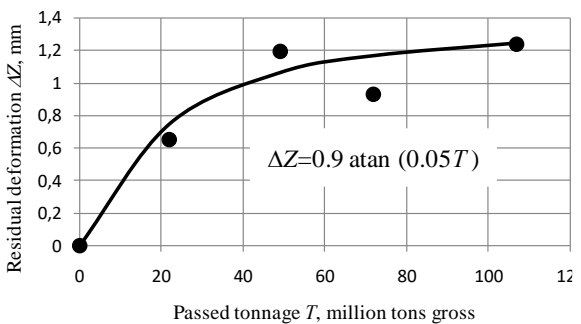

Figure 2

Influence of passed tonnage on horizontal residual deformation of the elastic clamp in straight section of track

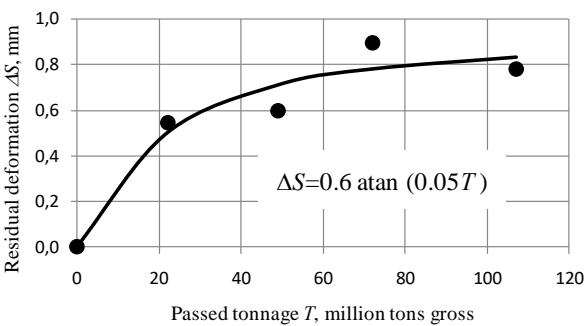

Figure 3

Influence of passed tonnage on vertical residual deformation of the elastic clamp

As the factor analysis showed, passed tonnage is a very influential factor. Here, it should be understood that passed tonnage is the sum of the loadings of the rolling stock axles. And the rolling stock moves through the track section for a certain period of time. While the pressure of the axles sums up, time goes by. Therefore, the passed tonnage influence on the elastic clamps is the effect of time under the rolling stock impact and the mounting forces of the clamps. Hence, it is necessary to observe the track of the object No. 2, which technical characteristics are similar to the technical characteristics of the track of the object No. 1, but the rolling stock impact is absent. Under this condition, it is possible to determine the time influence when only the mounting forces of the fastenings are acting. In this work, elastic fastenings that meet the requirements of regulatory and technical documentation were used. Thus, it is supposed that, at the beginning of the tests, the mounting forces of the clamps are the same.

The observation results of the horizontal residual deformation $\Delta Z$ of the elastic clamps of the object No. 2 are shown in Figure 4. The points on the graph show the average values of measurements at different times. The obtained data are approximated by the function $\Delta Z=E \cdot \operatorname{atan}(F \cdot t)$, where $E$ and $F$ are constant 
approximations: $E=0.9 ; \quad F=0.33$. Along the ordinate axis is the residual deformation $\Delta Z$. Time $t$ is on the abscissa.

The results show that the approximating functions in Figure 2 and Figure 4 are the same. It is interesting that $F=B \cdot 107 / 16$. The ratio $107 / 16$ is the track load intensity of the object No. 1 expressed in months (Table 1).

The observation results of the vertical residual deformation $\Delta \mathrm{S}$ of the elastic clamps of the object No. 2 are shown in Figure 5. The obtained data are approximated by the function $\Delta S=G \cdot \operatorname{atan}(H \cdot t)$, where $G$ and $H$ are constant approximations: $G=0.8 ; H=0.33$. On the y-axis - the residual deformation $\Delta S$. On the abscissa is time $t$.

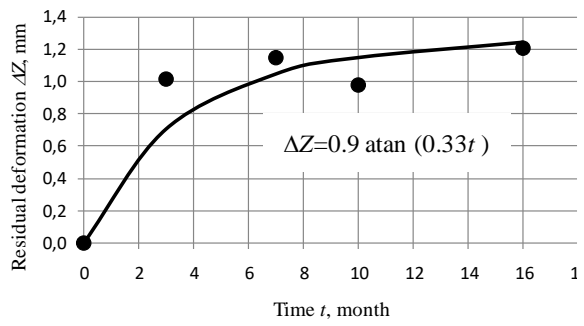

Figure 4

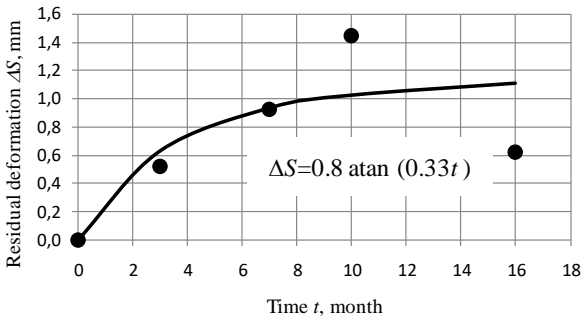

Figure 5

Influence of time on horizontal residual deformation of the elastic clamp

Influence of time on vertical residual deformation of the elastic clamp

The results show that the approximating functions in Figures 3 and 5 do not match. Approximation constant $H=D \cdot 107 / 16$. The ratio $107 / 16$ is the track load intensity of the object No. 1 expressed in months. It is interesting that the vertical residual deformation of the elastic clamps, where there was a movement of trains, is less. This is due to the fact that under the wheel, the under-rail pad is compressed, and the operating stresses in the clamp are periodically reduced. The relaxation is the higher, the higher the level of operating stress. [6]. Thus, the dynamic vertical forces of the track and rolling stock interaction reduce the vertical residual deformation.

Then it is obvious that in Figures 2 and 4, the approximating functions of the horizontal residual deformations coincide due to the lack of dynamic lateral forces in the straight lines. If this statement is true, then the graph of horizontal residual deformations $\Delta Z$ for the curve should have a larger amplitude compared to the graph in Figure 2 due to the influence of lateral horizontal forces [7, 8].

Figure 6 shows a graph of horizontal residual deformations $\Delta Z$ for a curve section with a radius of $874 \mathrm{~m}$. The obtained data are approximated by the function $\Delta Z=J \cdot \operatorname{atan}(K \cdot T)$, where $J$ and $K$ are constant approximations: $J=1.2 ; K=0.05$. Since $J>A$ then, indeed, the higher the relaxation, the greater the level of operating stresses. 


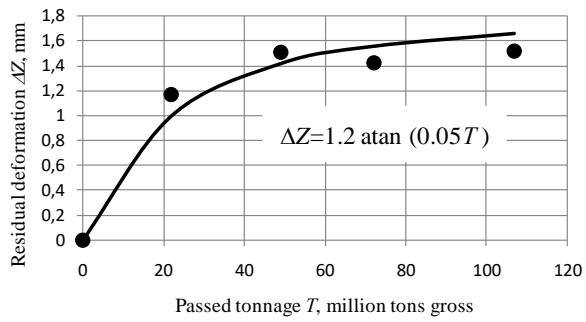

Figure 6

Influence of passed tonnage on horizontal residual deformation of clamp in curved section of track

As the factor analysis showed, the radius of the track has an effect, although insignificant, on the residual deformation of the elastic clamp. The combination of track radius and passed tonnage factors has a greater impact. Therefore, these track specifications of the object No. 3 and object No. 4 should be similar to those track specifications of the object No. 1. Under this condition, they can be compared with each other.

Further studies were performed by comparing sections of track with the same passed tonnage, the same curvature. The difference was in the operation time, there was an another load intensity.

On the track section of the object No. 3, the fastening measurements in the curved section with a radius of $745 \mathrm{~m}$ were performed. Passed tonnage was 49.7 million tons gross. Load intensity is 7.1 million tons gross/year/direction. Operation time is 7 years.

On the section of the track of the object No. 4, the fastening measurements in the curved section of the track with a radius of $885 \mathrm{~m}$ were performed. Passed tonnage was 46.9 million tons gross. Load intensity is 49.7 million tons gross/year/direction. Operation time is 0.9 years.

On the section of the track of the object No. 1, the fastening measurements in the curved section of the track with a radius of $874 \mathrm{~m}$ were performed. Passed tonnage was 46.1 million tons gross. Load intensity is 87.1 million tons gross/year/direction. Operation time is 0.5 years.

Table 5

The results of measuring the residual deformation of the elastic clamp at different sections of the track

\begin{tabular}{|c|c|c|c|c|}
\hline $\begin{array}{c}\text { Track } \\
\text { section }\end{array}$ & $\begin{array}{c}\text { Load intensity, million tons } \\
\text { gross/year/direction }\end{array}$ & $\Delta Z, \mathrm{~mm}$ & $\Delta S, \mathrm{~mm}$ & $\begin{array}{c}\text { Operation time, } \\
\text { years }\end{array}$ \\
\hline object No. 3 & 7.1 & 1.9 & 1.7 & 7.0 \\
\hline object No. 4 & 49.7 & 1.7 & 1.3 & 0.9 \\
\hline object No. 1 & 87.1 & 1.6 & 0.8 & 0.5 \\
\hline
\end{tabular}


Table 5 shows the results of measuring the residual deformation of the elastic clamp of the track sections with the same passed tonnage of $48 \pm 5 \%$ million tons gross, the same radius of $815 \pm 9 \% \mathrm{~m}$ and different load intensity.

\section{Results and Discussion}

The influence level of time on the residual deformation of the elastic clamp is estimated using one-factor analysis based on the measurements of the object No. 2. The elastic clamps at object No. 2 were measured five times. Therefore, five factor levels were adopted for one-factor analysis. Table 6 shows the results of one-factor analysis for the significance level $\alpha=0.05$. The results show that time is a very influential factor.

Table 6

One-factor analysis results of the time influence on the residual deformation of the elastic clamp

\begin{tabular}{|c|c|c|c|c|c|}
\hline \multicolumn{3}{|c|}{ for size "Z" } & \multicolumn{3}{c|}{ for size "S" } \\
\hline$F$ & {$[F]$} & conclusion & $F$ & {$[F]$} & conclusion \\
\hline 7.516 & 2.467 & affects & 16.803 & 2.467 & affects \\
\hline
\end{tabular}

Table 7 shows the results of determining the vertical residual deformation of the elastic clamp with a passed tonnage of 50 million tons gross and a different load intensity, obtained from the literature [9-11], describing the similar studies. The data in Table 7 are reduced to the passed tonnage of 50 million tons gross using the established functions of the influence of passed tonnage on the residual deformation of the elastic clamp.

Table 7

The results of determining the residual deformation of the elastic clamp according to literary sources

\begin{tabular}{|c|c|c|c|}
\hline Literary sources & $\begin{array}{c}\text { Load intensity, million tons } \\
\text { gross/year/direction }\end{array}$ & $\Delta S, \mathrm{~mm}$ & $\begin{array}{c}\text { Operating } \\
\text { time, years }\end{array}$ \\
\hline$[9]$ & 15 & 1.6 & 3.3 \\
\hline$[9]$ & 57 & 1.3 & 0.9 \\
\hline$[10],[11]$ & 211 & 0.5 & 0.2 \\
\hline
\end{tabular}

The data in Table 7 clearly confirm the conclusion of the one-factor analysis that the operating time affects the residual deformation of the elastic clamp. In addition, the data in Table 7 can be used as a supplement to the data in Table 5.

Figure 7 shows the effect of time with train load on the horizontal residual deformation of the elastic clamp for curved track sections with a radius of $815 \pm$ $9 \% \mathrm{~m}$. The vertical wear influence of under-rail pads and insulators on the horizontal residual deformation $\Delta Z$ is not established. Therefore, the data can be combined for different passed tonnages. The points on the graph show the data of 
Figure 6 and Table 5. The obtained data are approximated by the function $\Delta Z=P \cdot \operatorname{atan}(Q \cdot \tau)$, where $P$ and $Q$ are constant approximations: $P=J=1.2$; $Q=K \cdot 12 \cdot 107 / 16=4$. The ratio $12 \cdot 107 / 16$ is the track load of the object No. 1 expressed in years. On the $y$-axis is shown the residual deformation $\Delta Z$. On the abscissa axis - time with train load $\tau$. Time with train load is the ratio of passed tonnage to load intensity, that is $\tau=T /(12 \cdot 107 / 16)$.

Figure 8 shows the effect of time with train load on the vertical residual deformation $\Delta S$ of the elastic clamp for curved sections of track with a radius of $815 \pm 9 \% \mathrm{~m}$ and passed tonnage up to 50 million tons gross. The points on the graph show the data of Figure 8 (partially), Table 5 and Table 7 . The obtained data are approximated by the function $\Delta Z=U \cdot \operatorname{atan}(V \cdot \tau)$, where $U$ and $V$ are constant approximations: $U=L=1.0 ; V=M \cdot 12 \cdot 107 / 16=4$. On the y-axis is shown the residual deformation $\Delta S$. On the abscissa axis - time with train load $\tau$.

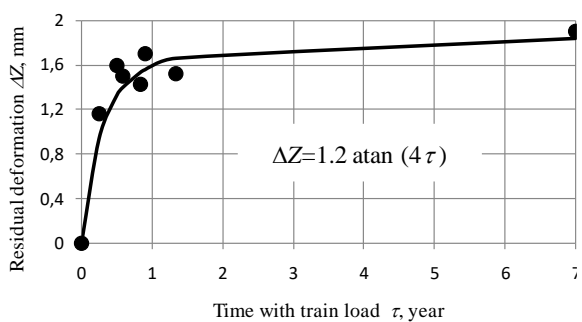

Figure 7

Time influence with train load on horizontal residual deformation of the elastic clamp

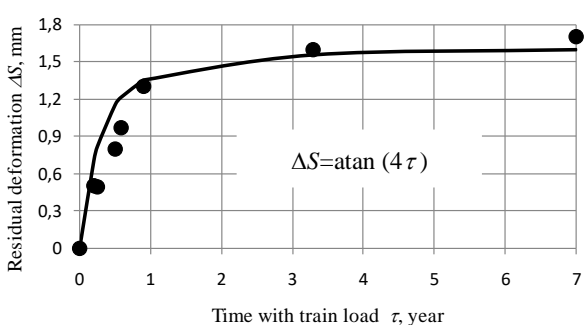

Figure 8

Time influence with train load on vertical residual deformation elastic clamp

\section{Conclusions}

According to the research results of the elastic clamp relaxation of the rail fastenings, the following conclusions can be made:

- there is a relaxation process in the elastic clamps of the rail fastening, which has a curvilinear dependence on time;

- the intensity of the elastic clamps relaxation attenuates after one year of operation;

- in addition to the time factor, the relaxation of elastic clamps is influenced by the level of operating stresses;

- greater value of operating stresses corresponds to greater relaxation;

- the influence of operating stresses is manifested as a factor of passed tonnage, a factor of the curve radius; 
- the factor of passed tonnage is expressed by the impact of vertical and horizontal forces: vertical force reduces relaxation by the pad compression and reduction of level of the elastic clamp working stresses;

- the factor of the curve radius is expressed by the action of horizontal forces that increase the relaxation of the elastic clamps by their additional deformation and increase the level of operating stresses;

- no effect on the relaxation of the mounting-dismantling operation of the elastic clamp was detected;

- the effect of temperature on the relaxation of the elastic clamp is not detected.

\section{References}

[1] M. Kurhan, D. Kurhan. The effectiveness evaluation of international railway transportation in the direction of "Ukraine - European Union", Transport Means, 2018, pp. 145-150

[2] Regulations on the procedure for conducting acceptance tests of prototypes of the elements of the upper structure of the track. CP-0139. - Kyiv, 2006. $39 \mathrm{p}$.

[3] A. K. Mitropolskiy. Technique of statistical computations. Ed. 2., "Science", M.: 1971. - 576 p.

[4] A. G. Bondar, Planning an experiment in chemical engineering. "Graduate school", K.:1976. - 184 p.

[5] S. V. Grachev, L. A. Maltseva. Stress relaxation of the spring tape during bending. GOU VPO "Ural State Technical University - UPI". 2005. - 18 p.

[6] V. P. Belogur, V. V. Voroshilin, G. A. Danilin. Investigation of the relaxation resistance of helical cylindrical springs during prolonged exposure under load. Metal working, No. 3, 2014, pp. 30-35

[7] S. Fischer. Comparison of railway track transition curves. Pollack Periodica, Vol. 4(3), 2009, pp. 99-110

[8] M. Kurhan, D. Kurhan, R. Novik, S. Baydak, N. Hmelevska. Improvement of the railway track efficiency by minimizing the rail wear in curves. IOP Conf. Ser.: Materials Science and Engineering, Vol. 985, 2020, 012001

[9] K. V. Moiseenko, V. M. Suslov, A. A. Taturevich. Justification of the need to abolish the restrictions on the use of fasteners type PPC- 5 by the criterion of load. Railway transport of Ukraine. 2/2017, -55 p.

[10] Conclusion on the results of field tests of intermediate rail fastenings KPP-5 and KPP-7. OJSC "Railway Transport Research Institute”, 2010

[11] Test protocol No. 2 gasket subrail type PRP-3.2 made of polyurethane based on polyesters Elastolan 90A. Institute of Ferrous Metallurgy of the National Academy of Sciences of Ukraine. Dnipro, 2016 